Ensayo Original / Original Essay

\title{
Gerencia Estratégica y Disrupción
}

\author{
Autor: Giovanni Felipe Torrealba Infante \\ Universidad Centroccidental "Lisandro Alvarado", UCLA \\ g torrealba@ucla.edu.ve \\ Lara, Venezuela \\ https://orcid.org/0000-0001-8760-3397
}

\section{Resumen}

El presente trabajo explora el impacto del desarrollo de las capacidades de virtualización y ubicuidad que aportan las tecnologías de información y comunicaciones (TIC's) con características disruptivas a los recientes enfoques de la gerencia estratégica. Las tecnologías modernas, en especial las de información y comunicaciones se han convertido en el recurso estándar para el ámbito gerencial en cualquier sector empresarial. Gracias a la diversidad de soluciones y aplicaciones que inundan la web, y a la facilidad para la incorporación de nuevas ideas al crecimiento y desarrollo de este conglomerado de herramientas para el manejo de información, se hace posible el repensar de la cotidianidad en la actividad gerencial, permitiendo la optimización de múltiples funciones ejecutivas. Es así como el gerente moderno, frente a nuevas o viejas oportunidades de acción empresarial, debe cuestionar las decisiones tradicionales y formularse en ese sentido mecanismos disruptivos guiados con la idea de que cada posibilidad tiene su especificación para el logro del éxito, en especial con el apoyo de múltiples soluciones automáticas puntuales.

Palabras clave: gerente; planificación estratégica; gestión; automatización; nuevas tecnologías. 


\section{Strategic Management and Disruption}

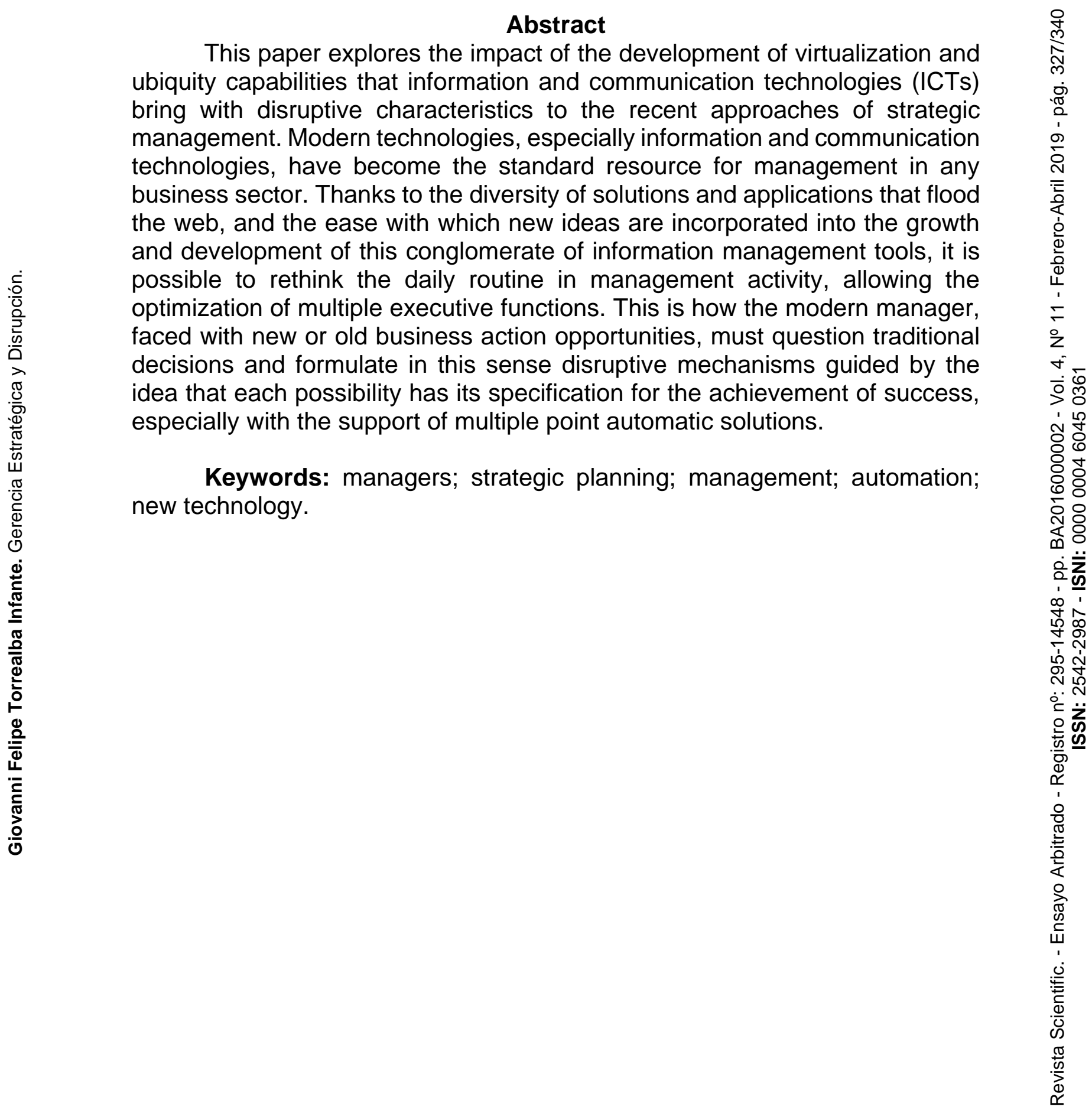

Date Received: 04-06-2018

Date Acceptance: 31-08-2018 


\section{Introducción}

Nuevos desafíos y oportunidades emergen de la utilización de nuevas tecnologías que implican necesariamente la revisión del quehacer gerencial tradicional, toda vez que día a día son mayores.

Determinar la ruta más eficiente para desarrollar la visión y la misión empresarial es para los directores ejecutivos en la actualidad, una permanente evaluación del cómo influir en su medio, que implica muchas veces rebelarse conscientemente al estatus quo para aprovechar oportunidades claves, utilizar fortalezas para disminuir el impacto de amenazas y vencer debilidades. Además, requiere mayor intuición respecto a las estrategias de competidores, mayor productividad del talento humano, menor resistencia al cambio. En sí, es el camino de formular los ¿cómo lograrlo?, dejando atrás el "descartemos esto". Allí está la disrupción.

En este contexto, resulta oportuna la frase de William G. McGowan, citado en (Pérez y Coutin, 2005, pág. 2): empresario estadounidense, fundador y presidente de $\mathrm{MCl}$ Communications Corp, "La única práctica gerencial que ahora es constante es la práctica de acomodarse constantemente al cambio", como muestra de la imperiosa necesidad de articular y adecuar acciones estratégicas que permitan alcanzar a la organización las metas propuestas.

\section{Pensamiento Disruptivo}

La teoría del pensamiento disruptivo plantea el desarrollo de nuevas estrategias o modelos de negocio a partir de las ideas que generamos y descartamos por considerarlas fuera de lo conocido. Se trata de "una forma de pensar que produce una estrategia poco convencional que deja a los competidores luchando por ponerse al día... que pone patas arriba las expectativas del consumidor y lleva a la industria a su próxima generación" (Luke, 2016a, pág. 3).

Es este orden de ideas, refiere el autor, la gerencia organizacional debe 
pensar diferente, desarrollando la capacidad de imaginar las cosas como jamás antes fueron, frente a un entorno actual cambiante, aprender a pensar y actuar de manera disruptiva, a fin de no ser sorprendidos por empresas emergentes que nos desplacen por lo innovador de sus productos o servicios alternativos.

Un reciente estudio sobre la Consultora (KPMG) realizado sobre disrupción y crecimiento afirma que "tres de cuatro de los directores ejecutivos (74\%) piensan que en lugar de esperar que surja una innovación disruptiva en la competencia, ellos se centran en generar disrupciones en el sector donde operan" (Goodburn, 2017, pág. 6). De igual manera se destaca en este estudio que un sesenta y cinco por ciento $(65 \%)$ percibe que disrupción tecnológica es más una oportunidad que una amenaza.

En la práctica, para las organizaciones consiste en una serie de pasos descritos como un proceso de cinco etapas a través de las cuales se desarrolla el pensamiento disruptivo, los cuales se titulan así:

Elaboración de una hipótesis disruptiva, definición de una oportunidad de mercado disruptiva, generación de varias ideas disruptivas, dar forma a las ideas en una solución disruptiva práctica y proponer la idea de manera que persuada a los interesados a invertir o adoptarla (Luke, 2016b, pág. 7).

\section{Virtualidad}

Las nuevas tecnologías desempeñan un papel determinante en esta era de cambios, en conjunto con la estrategia disruptiva; es así como los conceptos de virtualidad y ubicuidad, posibles gracias a la amplia gama de estos dispositivos (TIC`s), deben ser analizados. Sobre este respecto el estudio de un modelo base para el establecimiento de un sistema de gestión de apoyo a la estrategia organizativa, confirma el rol crítico del factor "uso de aplicaciones informáticas", considerándolo como "un elemento que apoya el potencial de éxito" (Torrealba y Lucena, 2018, pág. 170). 
La visualización de ubicuidad de Weiser establece que "la disposición de la tecnología puede funcionar de acuerdo con las necesidades de las personas sin requerir una atención continua, permitiendo que los objetos tecnológicos sean usados de manera natural, volcando la atención del sujeto hacia otros objetos" (Weiser, 1991, pág. 94). Según el autor, el poder de la ubicuidad no está en los dispositivos, si no en sus interacciones; para él, las computadoras personales serían una etapa de transición.

En particular, la virtualidad en las organizaciones se caracteriza por la separación por el espacio, tiempo y las fronteras organizativas de sus integrantes, por un eficaz y eficiente proceso de intercambio de conocimientos en las dimensiones de espacio, tiempo y estructura, estableciendo la presencia virtual, como una permanencia virtual y accediendo a capacidades virtuales.

\section{Gerencia Estratégica Virtual Disruptiva}

Mi planteamiento de la Gerencia Estratégica (GE) del proceso gerencial, se centra en el pensamiento disruptivo del equipo de alta dirección de la organización, y la transformación virtual de las organizaciones como eje central de la estrategia organizativa, y a las (TIC`s) como herramienta habilitadora de todos los procesos de planificación y visión del futuro.

Como elementos determinantes de la configuración de la (GE) se tienen: una estructura operacional de trabajo flexible para el aseguramiento de la calidad en la producción de bienes o servicios y la satisfacción del cliente, liderado por directores ejecutivos que practican un enfoque de Gestión Participativa del equipo de trabajo, y las (TIC`s) como elementos clave que integran los recursos, y constituyen el factor diferenciador de una estrategia adaptable.

La Gestión Participativa contribuye a mejorar el desempeño organizacional, con un sistema de administración o gestión basado en la participación activa de los integrantes de la organización, capitalizando los 
aportes y contribuciones de cada talento humano. Es así como la gestión participativa "forma parte de los elementos de la capacidad organizativa" y puede mejorar la eficacia de la organización, así como "contribuir al buen liderazgo e incrementar la transparencia en la toma de decisiones organizativas" (Bartle, 2007, párr. 48).

Este estilo de gestión de filosofía entiende a la organización "como un medio a través del cual el ser humano es dignificado y es desarrollado en todos los aspectos que lo hacen ser humano" (Arandia y Portales, 2015, pág. 140); acogiendo diversos valores que promueven la motivación del personal, logrando un mayor desempeño del recurso humano y por ende mayor productividad corporativa.

Lo anterior implica la integración del talento humano en la organización, con un fuerte compromiso con valores y principios de eficacia y eficiencia, utilizando el liderazgo participativo para trasladar áreas de decisión a los trabajadores. Asimismo, se señala que mediante este estilo de gestión se logra que el potencial humano de la organización este constituido por individuos empoderados y que poseen los conocimientos y herramientas para autogestionarse.

Sustentado en la Teoría Z, descrita como participativa y basada en las relaciones humanas, se considera que el empleo es parte esencial en la vida del trabajador, lo que le permite mantener un estilo de vida acorde a sus preferencias, definiendo su plan de jubilación, consolidando estrechas relaciones sociales a través de la confianza, fundamento de la cultura $Z$.

Por otra parte, el desarrollo de la competencia digital virtual del talento humano de las organizaciones es el factor determinante para el éxito de toda organización del siglo XXI. Ante un panorama de evaluación de la competitividad de la organización, las (TIC's) se convierten en un elemento clave para lograr la integración de los recursos.

Es así como, las (TIC`s) son actualmente el recurso para integrar, 
planificar y esbozar las estrategias de dicha organización, su uso tiene un impacto significativo ante acciones estratégicas y viabilidad operativa. Como rasgo destacado para el incremento del desempeño estratégico, se observa a las (TIC's) como una herramienta habilitadora de todos los procesos de planificación y visión del futuro, potenciados gracias a la posibilidad del trabajo a distancia y por ende la formación de equipos especializados y multidisciplinarios.

En este mismo sentido, las (TIC's) establecen la conexión organizacional del negocio con sus clientes y entorno donde se desarrollan las operaciones, y facilitan la interactividad entre las partes involucradas, proporcionando un valor agregado y social a la empresa.

Por otra parte, las (TIC's) también favorecen la creación de alianzas estratégicas a distancia que favorece la multidisciplinariedad de los integrantes del equipo de trabajo, adoptando una nueva concepción de trabajo a distancia, traspasando los límites corporativos de una manera globalizada.

Adicionalmente, la tercera evolución de las organizaciones responde a las organizaciones basadas en información; donde las Tecnologías de Información permiten responder a dos (02) elementos determinantes para la supervivencia en el mundo competitivo actual: un cambio en las expectativas de los clientes (productos orientados a necesidades individuales más que generales) y un cambio en la estructura de los mercados, que ahora es globalizado.

De esta manera, las (TIC`s) se consideran como un factor estratégico a nivel gerencial, en tanto que propician el crecimiento institucional y del recurso humano. Las organizaciones actuales, frente a estos impactos tecnológicos, están en la obligación de repensar el negocio, aplicando innovaciones disruptivas, tomando como pilares las marcadas tendencias que significan la computación en la nube, la movilidad, las redes sociales y la seguridad.

En particular, en las actividades de la cadena de valor de una empresa 
distingue claramente el componente físico y el de procesamiento de información; es decir, todas las actividades de valor crean y usan algún tipo de información. Por tanto, las (TIC`s) juegan un papel imprescindible a todo nivel de la empresa, desde quien desarrolla una idea, pasando por quien le da forma y quienes la llevan a cabo; la unión de todos los eslabones es una tarea que exige disponer de los mejores recursos tecnológicos disponibles, para el logro de los objetivos corporativos. Según esto, las Tecnologías de Información generan un impacto al posibilitar la recolección y análisis de información de todas las áreas de la organización, y de formas que antes no se disponían.

Es así como las (TIC's) brindan a la gerencia una herramienta de gestión competitiva que les permite permanecer o destacarse ante las modernas exigencias del mercado global, incluso coordinando actividades con localidades remotas y con nuevas redes de negocios, adecuando el planteamiento de innovadoras maneras de crear y gestionar proyectos, procesos y recursos con estrategias menos restrictivas frente a las distancias, volúmenes y velocidad de datos.

Sobre la base de todo este panorama, es necesario destacar la pertinencia de la incorporación de la informática ubicua. En palabras de Russel Ackoff, citado en (Agudelo, 2011, párr. 1): "comprender la naturaleza de los cambios de forma global y no parcial, para lo cual se debe desarrollar una mejor visión del mundo en concordancia con los nuevos métodos de la época"; de esta manera, en la medida que la informática ubicua cobre más auge en las empresas, se convertirá en una línea estratégica de desarrollo, y, en consideración del impacto que representa, su incorporación resultará en un componente de apoyo a la gerencia estratégica.

Es por lo que, el componente tecnológico ubicuo se convierte en una herramienta que apoya en el abordaje, evaluación y toma de decisiones por parte de un gerente que ocasionalmente estará físicamente fuera de la organización, y que además se anticipa y decide de una manera oportuna, 
clara y sencilla.

Según lo expresado, puede asegurarse que el componente tecnológico ubicuo será aprovechado como apoyo a la formulación de estrategias al momento de establecer debilidades, fortalezas, oportunidades y amenazas de factores externos a la organización. Las organizaciones actuales, frente a estos elementos de ubicuidad, no deben ignorar este factor de innovación y su potencial estratégico para la gerencia, puesto que la tendencia se orienta a fuentes de información ubicuas que nutrirán la analítica para la toma de decisiones.

Lo anteriormente planteado indica que las organizaciones actuales centran el desarrollo de sus estrategias operacionales en un entorno tecnológico caracterizado por la disponibilidad de innovadoras herramientas informáticas, que ponen a disposición de la gerencia la posibilidad de adoptar estrategias modernas, facilitando posturas y pensamientos disruptivos, en especial con un cambio de paradigma hacia escenarios donde la virtualidad y la ubicuidad juegan un papel determinante para la competitividad de la empresa.

Asimismo, la transformación virtual de las organizaciones requiere de directivos, gerentes o líderes capaces de revalorar la operación actual de su negocio a la luz de los nuevos avances que las (TIC's) imponen y convertir este nuevo quehacer empresarial mediado por las (TIC's) en un efectivo y decisivo factor diferenciador de una estrategia flexible y adaptable.

Esta innovación debe ser disruptiva, producto de un proceso gerencial que considere que pensar diferente y fuera de lo conocido es la base de innovaciones (innovaciones disruptivas). La gerencia estratégica moderna cuenta, por tanto, con elementos que le permiten alcanzar las ventajas competitivas frente al pujante mercado global que la circunscribe, y que además son denominadores en común en las pautas que marca la competencia. Es por tanto imperativo fomentar el desarrollo del pensamiento 
disruptivo de los miembros del equipo de alta dirección de la organización como factor decisivo.

El término disrupción se aplica en el contexto tecnológico "para referirse a productos o servicios tan revolucionarios que dejan obsoleto todo lo anterior en muy poco tiempo" (García, 2013, pág. 84). Más allá de este ámbito, ha comenzado a utilizarse para productos que no compiten en funcionalidades y calidad, pero son mucho más económicos y buenos para resolver problemas específicos.

En un estudio realizado sobre cómo los dispositivos móviles inteligentes modifican la forma en que las empresas generan valor para sus clientes, se describe el proceso de interacción y empoderamiento del cliente, denominado el círculo del valor digital, como "un proceso de interacción y empoderamiento en el que el cliente se encuentra en el centro de la agregación de valor" (Auletta, 2013, pág. 13); lo cual representa una trasformación radical de la forma en la que las empresas adaptan nuevas tecnologías y las incorporan a sus procesos de negocios.

La innovación tecnológica, la disrupción de procesos y la transformación de servicios, constituyen las tres fuerzas claves señaladas como "la revolución más reciente que ha experimentado la industria de servicios financieros" (Gomber, Kauffman, Parker y Weber, 2018a, pág. 221). Para los autores, la búsqueda de nuevas vías para modelos de negocios exitosos y la creación de una experiencia mejorada del cliente puede ser materializada mediante la disrupción impulsada por la plataforma tecnológica.

Los citados autores concluyen que la dinámica para el sector financiero actual, y que incluye muchos otros sectores, "implica evaluar las innovaciones, disrupciones y transformaciones provenientes de tecnologías emergentes, que les permita rediseñar procesos, incorporar nuevos productos amigables y valiosos e implementar cambios permanentes en los servicios" (Gomber et al., 2018b, pág. 223). 
En este orden de ideas, el desarrollo del pensamiento disruptivo implica fomentar la autotransformación, la experimentación como factor de aprendizaje, el conocimiento de fuentes de innovación y una permanente vigilia tecnológica. Es enfocar los problemas desde una perspectiva diferente a la tradicional, trabajar de forma radicalmente distinta, con métodos diferentes a los establecidos.

Es así que puede señalarse a las tecnologías como el canal que permite generar innovación radical, en comparación con el canal tradicional; en ese sentido se afirma que "la fuerza technology push (impulsar nuevos productos o tecnologías desde el interior de la organización, sin que lo pida el mercado) crea auténticos océanos azules" (Ferrás, 2014a, párr. 1). En estos planteamientos, se destaca a la innovación disruptiva impulsada por la tecnología, frente a los nuevos modelos de negocios, y se contrasta con la innovación inducida por el mercado, donde las demandas "difícilmente requerirán un cambio radical de paradigma, o un rediseño completo de la arquitectura dominante del producto o servicio" (Ferrás, 2014b, párr. 2).

\section{Consideraciones Finales}

El logro de ventajas competitivas diferenciadoras como resultado de un proceso de pensamiento gerencial disruptivo, en un contexto virtual ubicuo, dinámico y complejo, constituye el marco referencial de mi perspectiva gerencial. Esta perspectiva, se caracteriza por un cambio radical en la forma de pensar del gerente, limitado por ahora a seguir los modelos establecidos, y considera que el desarrollo de nuevas opciones a partir de ideas que lucen en un principio como irracionales.

El pensamiento disruptivo, base de las organizaciones orientadas a la innovación, estimula la generación de ideas sin juzgarlas, incentiva el análisis de tecnologías cuyo funcionamiento es radicalmente diferente a la forma en que se utiliza en la actualidad, y apertura espacios de reflexión para el análisis 
de métodos y estrategias alternativas a las ya establecidas.

La diversidad de herramientas tecnológicas emergentes e innovadoras disponibles, ofrecen a las organizaciones la posibilidad de expandir sus operaciones a escala mundial; donde la gerencia no está circunscrita al espacio físico delimitado por la organización. En particular, la virtualidad como alternativa estratégica de la organización, obtiene valor de la compleja red de relaciones que emerge de la implementación de nuevas plataformas tecnológicas centradas en la ubicuidad.

\section{Referencias}

Agudelo, G. (2011). Modelos de Gerencia Estratégica. Blog. [Documento en línea]. Recuperado de:

https://gustavo-agudelo-velez.webnode.es/blog/gerencia/gerenciaestrategica/conceptos-basicos-gerencia/modelos-de-gerenciaestrategica

Arandia, O., \& Portales, L. (2015). Fundamentos de la gestión humanista: una perspectiva filosófica. $A D$-minister, (26), 123-147, ISSN: 16920279. Recuperado de:

http://www.redalyc.org/articulo.oa?id=322339789006

Auletta, N. (2013). Móvil: El impacto de los dispositivos personales en los negocios. Debates IESA, XVIII(3), 12-16, ISSN: 1316-1296.

Bartle, P. (2007). Gestión participativa: Métodos para incrementar la aportación del personal en la toma de decisiones organizativas. Canadá, Estados Unidos: Red Comunitaria de Vancouver, Colectivo de Potenciación Comunitaria, CEC. Recuperado de: http://cec.vcn.bc.ca/mpfc/modules/pm-pms.htm

Ferrás, X. (2014a,b). 11 claves para la innovación disruptiva. España: Sintetia. Recuperado de: http://www.sintetia.com/11-claves-para-la-innovacion-disruptiva/ 
García, C. (2013). Disrupción: la ciencia ficción llega a los mercados. Revista de Negocios del IEEM, 16(4), 84-86, ISSN: 2301-1173.

Gomber, P., Kauffman, R., Parker, C., \& Weber, B. (2018a,b). On the Fintech Revolution: Interpreting the Forces of Innovation, Disruption, and Transformation in Financial Services. Journal of Management Information Systems, 35(1), 220-265, ISSN: 1557-928X.

Goodburn, M. (2017). Disrupt and grow. Ámsterdam: KPMG International, 134512-G, 6-9. [Documento en línea]. Recuperado de:

https://assets.kpmg.com/content/dam/kpmg/xx/pdf/2017/06/2017global-ceo-outlook.pdf

Luke, W. (2016a,b). Disrupt: Think the unthinkable to spark transformation in your business. New Jersey, United States of America: Pearson Education, Inc.

Pérez, Y., \& Coutín, A. (2005). La gestión del conocimiento: un nuevo enfoque en la gestión empresarial. ACIMED, 13(6), 1-74. Versión impresa ISSN: 1024-9435. Recuperado de:

http://scielo.sld.cu/scielo.php?script=sci arttext\&pid=S1024$\underline{94352005000600004}$

Torrealba, G., \& Lucena, Y. (2018). Un Acercamiento a la Realidad: Iniciativas de Adopción del Cuadro de Mando Integral en Venezuela. Revista Scientific, 3(7), 155-176. Recuperado de:

https://doi.org/10.29394/Scientific.issn.2542-2987.2018.3.7.8.155-176

Weiser, M. (1991). The Computer for the 21st Century. Scientific American, 94-104. París, Francia: Université Paris-Sud, Laboratoire de Recherche en Informatique. Recuperado de:

https://www.Iri.fr/ mbl/Stanford/CS477/papers/Weiser-SciAm.pdf 


\section{Giovanni Felipe Torrealba Infante}

e-mail: g torrealba@ucla.edu.ve

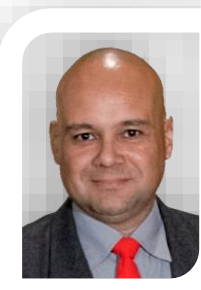

Nacido en Venezuela. Doctor en Gerencia Avanzada (UFT); Magíster Scientiarum en Sistemas de Información (UCLA); Ingeniero en Informática (UCLA); Docente ordinario en pregrado y postgrado en la Universidad Centroccidental "Lisandro Alvarado" (UCLA); Diplomado en Componente Docente (UCLA); Diplomado en Componente Docente en Educación a Distancia, Diplomado en Gerencia y Liderazgo. Empresario independiente, corresponsable del desarrollo y puesta en marcha de varios proyectos en las áreas de Sistemas de Información, bases de datos, aplicaciones móviles y portales web dinámicos.

El contenido de este manuscrito se difunde bajo una Licencia de Creative Commons ReconocimientoNoComercial-Compartirlgual 4.0 Internacional 